\title{
Multiple stellar populations in the massive clusters M22 and Omega Centauri
}

\author{
A. F. Marino \\ Max-Planck-Institut für Astrophysik, Karl-Schwarzschild-Str. 1, 85748 Garching, Germany \\ email: amarino@MPA-Garching.MPG.DE
}

\begin{abstract}
An intriguing discovery in the field of multiple stellar populations in globular clusters is that some of them show internal variations in the bulk of the heavy-element content. I summarize the chemical properties of one of these clusters, M22, in comparison with the most extreme $\omega$ Centauri, underlying the analogies and differences between the two objects.
\end{abstract}

Keywords. stars: abundances, globular clusters: individual (M22, $\omega$ Centauri).

\section{Introduction}

Since the '80s we know that light elements show peculiar patterns in globular clusters (GCs). On the other hand, variations in heavier elements were considered to be a trait of more massive systems capable to retain $\mathrm{SN}$ fast ejecta. In this respect the most massive Galactic GC $\omega$ Cen was always considered a peculiarity. In fact, to account for its well known huge metallicity variations, it has even been suggested that $\omega$ Cen is the remnant of a tidally disrupted dwarf galaxy rather than a real GC.

Surprisingly, recent discoveries have revealed that some GCs, besides $\omega$ Cen, have variations also in the bulk heavy element content, in analogy with more massive systems. Differently from the simple normal GCs that do not show evidence for SN-based self enrichment, in these objects successive generation(s) may need to be invoked, with $\mathrm{SNe}$ also playing a role in the pollution of intra-cluster medium.

Among these anomalous GCs, M22 is the first discovered (Marino et al. 2009, hereafter M09; Da Costa et al. 2009) and surely the one whose spectroscopic features more closely resemble $\omega$ Cen (Da Costa \& Marino 2011).

\section{M22 versus $\omega$ Centauri}

The most striking similarity between M22 and $\omega$ Cen is the internal variation in the overall metallicity. In M22, M09 and Marino et al. (2011a, hereafter M11a) obtain a total metallicity spread of more than a factor of two: $-1.97 \leqslant[\mathrm{Fe} / \mathrm{H}] \leqslant-1.57$, a range that cannot be explained by observational uncertainties. Note that, although metallicity variations are present in both clusters, in $\omega$ Cen the range in $[\mathrm{Fe} / \mathrm{H}]$ is more than a factor of 20 larger, with stars from $[\mathrm{Fe} / \mathrm{H}] \approx-1.90$ to $[\mathrm{Fe} / \mathrm{H}] \approx-0.60$.

In addition to this, the abundance distribution for elements mainly produced in the slow $(s)$ neutron-capture processes is clearly bimodal in M22 (M09, M11a). The two stellar groups with different $s$ element content ( $s$-rich and $s$-poor groups) are also characterized by: (ii) a mean different metallicity (M09); (ii) a mean different $\mathrm{C}+\mathrm{N}+\mathrm{O}$ content (M11a, Alves-Brito et al. 2012); (iii) in both groups internal variations in elements involved in the high temperature H-burning are present (M11a), so that each group individually traces the (anti)correlations in light elements found in normal GCs; (iv) on 


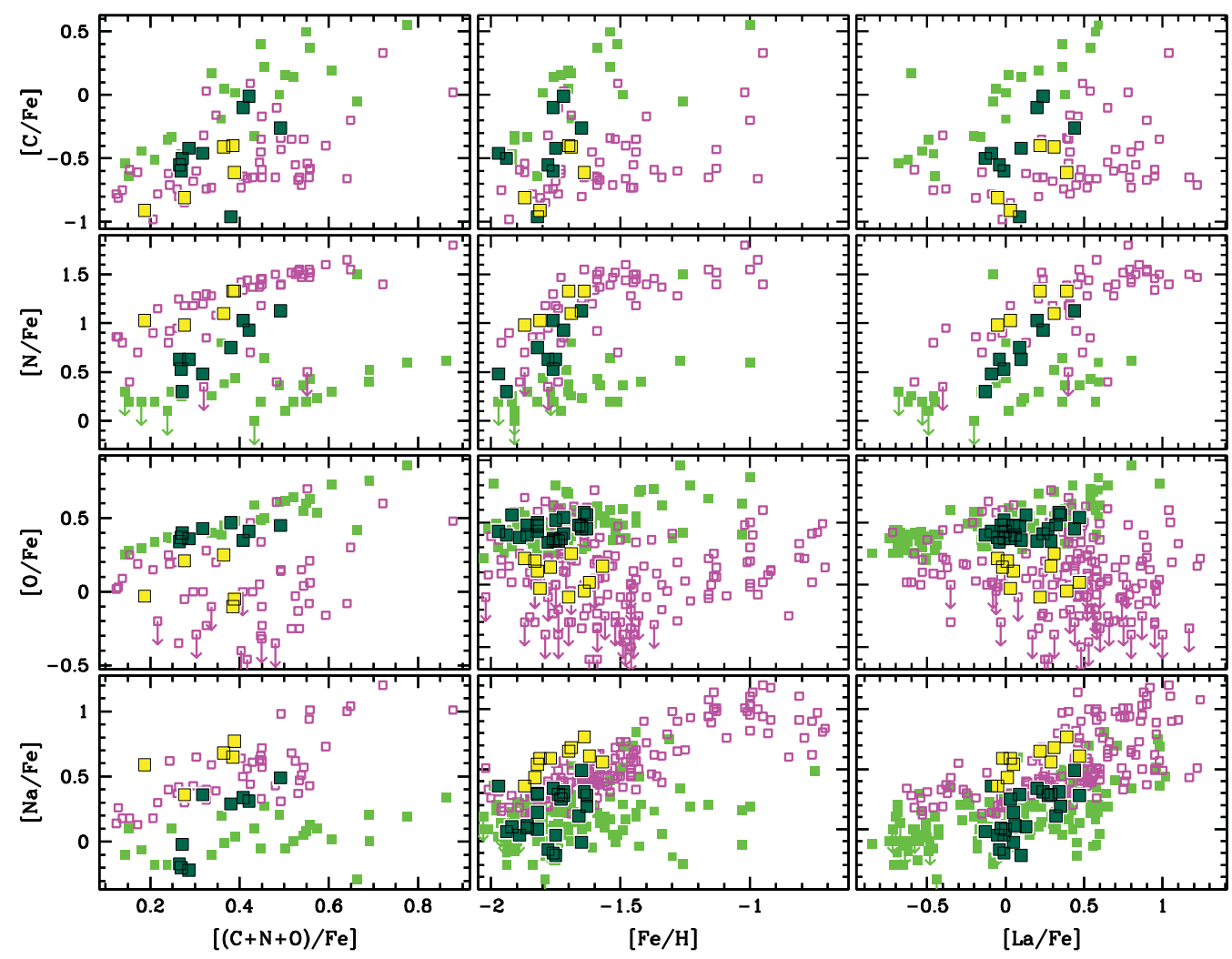

Figure 1. C, N, O Na relative to $\mathrm{Fe}$ as a function of $[\mathrm{CNO} / \mathrm{Fe}],[\mathrm{Fe} / \mathrm{H}]$, and $[\mathrm{La} / \mathrm{Fe}]$ for $\mathrm{M} 22$ and $\omega$ Cen stars. Dark-green and yellow points are the Na-poor and Na-rich stars in M22, and light-green and magenta points are the Na-poor and Na-rich stars in $\omega$ Cen, respectively.

the photometric side, M22 shows a split sub-giant branch (Piotto et al. 2012), whose sequences correspond to the two $s$ groups (Marino et al. 2012a).

The properties observed in M22 are also present in $\omega$ Cen, but again, in this latter they are much more extreme. In Figure 1 a collection of chemical abundances for M22 (from M09 and M11a) and $\omega$ Cen (Marino et al. 2011b, 2012b, hereafter M11b, M12b) is shown. In both clusters, stars have been divided into two groups on the basis of their position along the $\mathrm{O}-\mathrm{Na}$ anti-correlation (see M12b for details). Note that the separation of different stellar groups on the basis of their light-element abundances is just a possibility. Alternatively, the separation in stellar groups could be explored on the basis of metallicity or $s$ content (as in M11a, and M11b). An inspection of Figure 1 immediately gives an idea of how more extreme are the chemical variations in $\omega$ Cen, and, at the same time, how similar are these objects in terms of chemical patterns: ( $i$ ) all the $p$-capture elements (on the y-axis) have similar trends as a function of the CNO, Fe and $s$ element La; (ii) Na-poor and Na-rich stars separately show similar patterns in the two clusters.

The most natural development of these findings is to understand where these objects formed. The analogies with $\omega$ Cen, considered the possible relict of a dwarf galaxy, suggest the fascinating idea that also M22 could be the surviving nuclei of more massive system. 


\section{References}

Alves-Brito, A., Yong, D., Meléndez, J., Vásquez, S., \& Karakas, A. I., 2012, A 6 A, 540, A3

Da Costa, G. S., Held, E. V., Saviane, I., \& Gullieuszik, M., 2009, ApJ, 705, 1481

Da Costa, G. S., \& Marino, A. F., 2011, PASA, 28, 28

Marino, A. F., Milone, A. P., Piotto, G., et al., 2009, A\&A, 505, 1099

Marino, A. F., Sneden, C., Kraft, R. P., et al., 2011a, A\& A, 532, A8

Marino, A. F., Milone, A. P., Piotto, G., et al., 2011b, ApJ, 731, 64

Marino, A. F., Milone, A. P., Sneden, C., et al. 2012a, A 6 A, 541, A15

Marino, A. F., Milone, A. P., Piotto, G., et al. 2012b, ApJ, 746, 14

Piotto, G., Milone, A. P., Anderson, J., et al., 2012, arXiv:1208.1873 